\title{
Spectroelectrochemical Properties of Ultra-Thin Indium Tin Oxide Films under Electric Potential Modulation
}

\author{
Xue Han ${ }^{a} *$ and Sergio B. Mendes ${ }^{b}$
}

\author{
Department of Physics and Astronomy \\ University of Louisville \\ Louisville, KY, 40208
}

a: x0han004@louisville.edu; phone: (502)-5103917

b: sbmend01@louisville.edu; phone: (502)-852-0908, fax: (502)-852-0742

Abstract:

In this work, the spectroscopic properties of ultra-thin ITO films are characterized under an applied electric potential modulation. To detect minute spectroscopic features, the ultra-thin ITO film was coated over an extremely sensitive single-mode integrated optical waveguide, which provided a long pathlength with more than adequate sensitivity for optical interrogation of the ultra-thin film. Experimental configurations with broadband light and several laser lines at different modulation schemes of an applied electric potential were utilized to elucidate the nature of intrinsic changes. The imaginary component of the refractive index (absorption coefficient) of the ultra-thin ITO film is unequivocally shown to have a dependence on the applied potential and the profile of this dependence changes substantially even for wavelengths inside a small spectral 
window $(500-600 \mathrm{~nm})$. The characterization technique and the data reported here can be crucial to several applications of the ITO material as a transparent conductive electrode, as for example in spectroelectrochemical investigations of surface-confined redox species.

Keywords: indium tin oxide, spectroelectrochemistry, cyclic voltammetry, AC impedance potential modulation, integrated optical waveguide, absorbance spectroscopy

\section{Introduction}

Because of its good optical transparency and electrical conductivity, films of indium tin oxide (ITO) have been widely used in solar cells [1-3], displays [4, 5], electrochromic devices [6, 7], LEDs [8-10], and spectroelectrochemical applications [11, 12]. ITO films in the thickness range of about one hundred nanometer to one micrometer that had been grown by different deposition techniques and treated under diverse annealing conditions have been extensively investigated [13-16]. The conductivity and transparency of these films, and their relationship to the film crystalline structure have been widely studied [13]. However, those studies have been implemented for relatively thick ITO films (above $100 \mathrm{~nm}$ ) because it becomes particularly challenging to characterize the spectroscopic features of thinner (e.g., below $30 \mathrm{~nm}$ ) ITO films. The optical transparency and spectroscopic features of very thin ITO films cannot be precisely addressed by conventional transmission measurements and advanced characterization techniques are required in these cases. Another important limitation has been the lack of information on how the spectroscopic properties of the ITO film behave under potential modulations, which is crucial to understand the performance of those films at working conditions in a variety of applications. Only a few studies have been reported on the electrical properties of ITO films 
under potential modulations in different solution and electrolyte conditions [17-20], and on the refractive index of ITO films under potential modulation [21, 22], however those studies were limited to relatively thick ITO films.

In this present work, we apply an integrated optical waveguide technique to study the spectroscopic optical characteristics of ultra-thin ITO films. A planar, single-mode, integrated optical waveguide (IOW) creates a highly sensitive platform to investigate the spectroscopic properties of an overcoated ultra-thin ITO film. We report here investigations on the spectroscopic properties of ultra-thin ITO films performed under aqueous environment and different electric potential modulation approaches, which represent the working conditions present in many of the applications of ITO films. It is expected that the electrical modulation and environmental conditions will have a stronger impact in the properties of thinner films, as opposed to the thicker ones, and we aimed to address those effects in this work. Understanding the spectroscopic features of ultra-thin ITO films under those conditions is particularly critical for spectroelectrochemical and electro-optical applications that utilize an ITO film as a transparent working electrode [23].

\section{Thick ITO Films}

\subsection{Sample Preparation}

The ITO fabrication process was first optimized to create films with extremely low optical attenuation and good electrical conductivity. For this purpose, relatively thicker ITO films (ca. $400 \mathrm{~nm}$ ) were deposited on plain glass slides to identify the best fabrication conditions. Once those conditions were established, such protocol was then applied to deposit ultra-thin ITO films (ca. $13 \mathrm{~nm}$ ) over single-mode IOW devices described in Section 3. 
A pulsed-DC sputtering technique was used to deposit ITO films from a 3-inch target of $\left(\mathrm{In}_{2} \mathrm{O}_{3}\right) 90:(\mathrm{SnO}) 10$ wt $\%$ and $99.99 \%$ purity. The power of the pulsed-DC sputtering was set at $200 \mathrm{~W}$ with a frequency of $20 \mathrm{KHz}$ and a pulse duration of $1 \mu \mathrm{s}$. The substrates were kept at room temperature during deposition and the rotation speed of the substrate table was set at 20 r.p.m. to achieve uniform films. The flow rate of Ar was fixed at $12 \mathrm{sccm}$ and different flow rates of $\mathrm{O}_{2}$ were explored $(0.4 \mathrm{sccm}$ to $0.8 \mathrm{sccm})$ to optimize the electrical and optical properties of the ITO film. For each $\mathrm{O}_{2}$ flow rate, a 30-minute deposition process was used to grow a film with a thickness of approximately $400 \mathrm{~nm}$. After the deposition process, an inert annealing treatment in $\mathrm{N}_{2}$ atmosphere at $250^{\circ} \mathrm{C}$ for 10 minutes was used to activate $\mathrm{Sn}$ element, create additional oxygen vacancies, and minimize grain boundary effects to obtain lower resistivity and better transparency in the visible range [24].

\subsection{Optical and Electrical Properties}

Transmittance spectra of the thicker ITO samples were measured by a conventional spectrophotometer (Cary 300, Varian). An envelope technique [25] based on the transmittance spectra was employed to determine the thickness and refractive index (both real and imaginary parts) of the ITO films. The sheet resistance and thickness of the film were then used to calculate the film resistivity. In Figure 1 (a) and (b), the extinction coefficient (imaginary part of the refractive index) at the $550 \mathrm{~nm}$ wavelength and the resistivity are shown against the $\mathrm{O}_{2}$ flow rate during the sputtering deposition process. And those properties are compared for samples as sputtered and after the inert annealing treatment.

For the optical performance of samples before the inert annealing process, we observe in

Figure 1 (a) that the extinction coefficient decreases as the $\mathrm{O}_{2}$ flow rate increases from 0.40 to $0.65 \mathrm{sccm}$, and remains approximately constant above $0.65 \mathrm{sccm}$. The inert annealing process 
substantially reduces the extinction coefficient for samples deposited under low $\mathrm{O}_{2}$ flow rate (i.e. high values of the extinction coefficients), while the minimum extinction coefficient was found with an $\mathrm{O}_{2}$ flow rate of about $0.6 \mathrm{sccm}$. For the electrical resistivity (see Figure $1 \mathrm{~b}$ ), we notice that regardless of the $\mathrm{O}_{2}$ flow rate during deposition the inert annealing process consistently improves the electrical conductivity of the film probably by minimizing grain boundary defects in the films [26]. However, when the oxygen flow rate increases, the electrical performance of annealed samples shows a monotonic increase in their resistivity. A flow rate of $0.6 \mathrm{sccm}$ for the $\mathrm{O}_{2}$ gas during the sputtering process to deposit ultra-thin ITO films was established based on these measurements.

\section{Ultra-Thin ITO Films}

\subsection{Sample Preparation}

Planar, single-mode, integrated optical waveguide platforms have been used in challenging studies of adsorbed molecular thin films [27-30] due to their long effective pathlength. The strong optical interaction of a guided mode with a surface-adsorbed molecular assembly has enabled highly sensitive studies of weakly absorbing structures. Figure 2 schematically shows the IOW structure employed in this work to investigate the spectroscopic properties of ultra-thin films of the ITO material. Two surface-relief gratings $(25.4 \mathrm{~mm}$ apart from each other) with a pitch size of $323 \mathrm{~nm}$ were initially fabricated on glass substrates $(75 \times 25$ $\mathrm{mm}$ ) to work as integrated waveguide couplers. A highly transparent, single-mode, planar IOW device was created on those glass slides by depositing a 400-nm layer of $\underline{\mathrm{Al}}_{2} \underline{\mathrm{O}}_{3}$ and a $16-\mathrm{nm}$ layer of $\underline{\mathrm{SiO}}_{2}$ using an atomic layer deposition (ALD) process. Details of this fabrication process have been reported by us in the literature [30]. 
Based on the experimental results described in Section 2 for the (relatively) thicker ITO films, a flow rate of $0.6 \mathrm{sccm}$ for the $\mathrm{O}_{2}$ gas was selected to deposit a $13-\mathrm{nm}$ ITO film over single-mode IOW devices. After deposition, the same inert annealing process (previously described) was carried out for the ultra-thin ITO films, which provided films with consistently low resistivity values $\left(\rho=2.3 \times 10^{-3} \Omega \mathrm{cm}\right)$. However, the extinction coefficient did not decline to the expected value $\left(k=1.7 \times 10^{-4}\right)$ and an annealing process in the presence of oxygen (called here reactive annealing) was added to increase the optical transparency of the ultra-thin ITO films in the visible region. By annealing the samples in the presence of atmospheric oxygen, it is expected that oxygen vacancies in the film to decrease leading to a more complete stoichiometry in the ITO film. The fewer defects in the film should translate into lower residual absorption, and thus lower propagation losses for the waveguide modes. We started the reactive annealing process at $150{ }^{\circ} \mathrm{C}$ and, when the optical performance was still insufficient, the temperature was raised by increments of $50^{\circ} \mathrm{C}$. A sample was heated until an acceptable optical loss for the IOW device overcoated with an ultra-thin ITO film was achieved. With this reactive annealing process, a total propagation loss of as low as $6 \mathrm{~dB} / \mathrm{cm}$ was reached for the whole waveguide stack, which includes the alumina and silica films and the ultra-thin ITO film. The sheet resistance was about $2 \mathrm{~K} \Omega /$, which consistently corresponds to a value of $2.6 \times 10^{-3} \Omega \mathrm{cm}$ for the resistivity of the ITO film.

\subsection{Spectroelectrochemical flowcell}

Samples with low optical losses were placed in a buffer solution $\left(\mathrm{Na}_{2} \mathrm{PO}_{4}, 5 \mathrm{mM}, \mathrm{pH} 7\right)$ for at least 24 hours to chemically stabilize the ITO film. Then each sample was rinsed with DI water, dried by $\mathrm{N}_{2}$ gas, and set for electrical connections. A piece of carbon tape was used to 
attach a platinum wire onto the ITO film for electrical connection to the potentiostat (CHI 600D). A layer of silver paste was placed around the two gratings to provide a uniform electric potential for the whole ITO film. After the silver paste became dry, an insulating epoxy layer was placed over it to prevent any chemical reaction between the silver paste and the working electrolyte solution. After the epoxy layer was cured, the insulation between the epoxy layer and the platinum wire was tested, as well as the conduction between the platinum wire and the ITO film, which serves as the working electrode in our experiments. A homemade pseudo-reference electrode of $\mathrm{Ag} / \mathrm{AgCl}$ and a platinum counter electrode were inserted through the flow cell cover. The three electrodes were then connected to the potentiostat. The homemade pseudo-reference electrode in our electrolyte solution had a fairly constant potential offset of $85 \mathrm{mV}$ compared to a standard $\mathrm{Ag} / \mathrm{AgCl}$ reference electrode in $1 \mathrm{M} \mathrm{KCl}$ solution $(\mathrm{CHI})$. The potential values reported in this work are referenced to our pseudo $\mathrm{Ag} / \mathrm{AgCl}$ reference electrode.

\subsection{Cyclic voltammetry technique}

At the first usage in the electrochemical cell, the ultra-thin ITO film was further stabilized by performing a few scans of cyclic voltammetry $(\mathrm{CV})$ from $-0.4 \mathrm{~V}$ to $+0.8 \mathrm{~V}$ with various scan speed $(0.02 \mathrm{~V} / \mathrm{s}, 0.1 \mathrm{~V} / \mathrm{s}$ and $0.2 \mathrm{~V} / \mathrm{s})$ until the current signal became stable.

A tungsten-halogen lamp was employed as a broadband light source. The TE polarization was selected by placing a sheet polarizer in the optical path of the incoming beam before arriving at the grating coupler. Additional details of the optical coupling configuration can be found in elsewhere [31]. The out-coupled optical signal was collected by an optical fiber and delivered to a spectrometer that was connected to an intensified CCD (ICCD, Princeton, PIMAX 3). The out-coupled optical signal under CV potential modulation from $-0.4 \mathrm{~V}$ to $+0.8 \mathrm{~V}$ 
at a scan-speed of $0.02 \mathrm{~V} / \mathrm{s}$ was collected. The ICCD was set to an acquisition frequency of $2 \mathrm{~Hz}$ with having an exposure time of $200 \mathrm{~ms}$ for each frame.

On completion of these measurements, a trace corresponding to a dark signal was collected by injecting black ink into the cell and performing an acquisition under the same exposure time. Figure 3 shows the out-coupled broadband power spectrum at different values of the electric potential during a particular CV scan.

From the measurements above we calculated the normalized transmittance, $t_{N}$, according to Equation (1) for a particular wavelength:

$$
t_{N}(\lambda)=\frac{I_{V}-I_{\min }}{I_{\max }-I_{\min }}
$$

where $I_{V}$ is the out-coupled intensity (ICCD counts) at the electric potential $V$, and $I_{\min }$ and $I_{\max }$ are respectively the minimum and maximum out-coupled intensities at a particular wavelength when the potential was scanned from $+0.8 \mathrm{~V}$ to $-0.4 \mathrm{~V}$ over a few cycles. The traces of $t_{N}$ for a set of discrete wavelengths from $510 \mathrm{~nm}$ to $600 \mathrm{~nm}$ are shown in Figure 4. Each wavelength is vertically-shifted by one unit for clarity, and the several cycles of the CV scans are folded into one segment. For a particular wavelength, we observe that the folded traces demonstrate very good repeatability and consistency for the results during the CV scans. We also observe that different wavelengths have clearly distinct response to the same potential modulation scan. For example, at $600 \mathrm{~nm}$, the potential of lowest transparency is approximately at $-0.4 \mathrm{~V}$, but at 510 $\mathrm{nm}$ the potential of lowest transparency is at $+0.8 \mathrm{~V}$. And for $550 \mathrm{~nm}$, the transmittance shows a peak at about $+0.2 \mathrm{~V}$. 
In the measurements above a light source with a wide spectrum and a broadband waveguide coupler [31] were employed, and the coupling configuration was fixed at a particular geometry to give a band of about $100 \mathrm{~nm}$ centered at $560 \mathrm{~nm}$ (see Figure 3). Next, the same protocol of $\mathrm{CV}$ potential scans was sequentially applied to several monochromatic laser lines. However, for these following measurements, the flow cell was mounted on a rotation stage so that the incident angle could be adjusted to maximize the coupled light inside the waveguide for each laser line at open circuit potential. The normalized out-coupled transmittance against time (while the applied potential was scanned) is plotted in Figure 5, as well as the trace for the CV potential scan.

For wavelengths longer than $600 \mathrm{~nm}$, the transmittance follows the potential scan, i.e. they increase and decrease together. At $502 \mathrm{~nm}$, the transmittance and the potential scan change in opposite directions. The normalized transmittance curve at $543 \mathrm{~nm}$ shows both behaviors depending on the value of the potential. The folded 5-cycle of normalized transmittance against potential for each laser beam is shown in Figure 6.

Our measured data show very good consistent between the results obtained with broadband light at a fixed angle (Figure 3 and 4) and laser lines at their angle of maximum guided light (Figures 5 and 6). The consistency of these results above shows a clear dependence of the out-coupled optical light intensity on the applied electric potential and suggests two possible lines of explanation. One can hypothesize that the applied potential can either $i$ ) change the effective refractive index of the IOW device leading to a change in the coupling efficiency or ii) change the extinction coefficient of the ITO film leading to a change in the propagation losses. The elucidation of this conundrum is addressed in the next Section, where the first hypothesis was experimentally investigated with measurements of the out-coupled optical intensity against 
the incident angle for a series of laser lines and at several fixed values of the electric potential to determine if changes in the effective refractive index (due to changes in the applied potential) play an important role in the observed results.

\subsection{Potential steps technique}

Measurements were taken for several laser lines $(502,514,543,594$, and $633 \mathrm{~nm})$ at the open circuit condition and for electric potential values of $-0.4,-0.2,0.0,+0.2,+0.4,+0.6$, and $+0.8 \mathrm{~V}$. A polarizer was placed in front of the laser to select the TE polarization, and an achromatic doublet (Thorlabs AC 254-400-A1) with a long effective focal length was employed to collimate the laser beam. The flow cell was mounted on a rotation stage, which allowed us to precisely control the incident angle as we monitor the out-coupled optical intensity at a particular applied potential. For each laser line, we first acquired intensity measurements against the incident angle for the open circuit condition, and then sequentially repeated those measurements at each specific electric potential step listed above. Data for the out-coupled intensity against the incident angle for the 514 and $594 \mathrm{~nm}$ laser lines are shown in Figure 7 (a) and (b). We observe in those plots that, for a particular wavelength, the incident coupling angle for the maximum intensity is almost independent of the applied potential. However, at a particular angle of incidence, the intensity shows drastic changes with the applied potential steps. For the $594 \mathrm{~nm}$ laser line, the maximum out-coupled intensity at $+0.8 \mathrm{~V}$ potential is more than 6 times larger than the maximum out-coupled intensity at $-0.4 \mathrm{~V}$ potential, although the coupling angle is about the same $\left(13.45^{\circ}\right)$. For $514 \mathrm{~nm}$ laser line, the maximum out-coupled intensity at $-0.4 \mathrm{~V}$ potential is almost 5 times higher than the maximum out-coupled intensity at $+0.8 \mathrm{~V}$ potential. 
By using the incident angle that maximizes the coupled power for each applied potential, the effective refractive index $N_{\text {eff }}$ can be calculated as described in Equation (2), where $n_{i n}$ is the refractive index of the incident media (air, in this case), $\theta_{i n}$ is the coupling angle, $\lambda$ is the wavelength of the laser line, and $\Lambda$ is the pitch size of the grating ( $323 \mathrm{~nm}$ ).

$$
N_{\text {eff }}=n_{\text {in }} \sin \theta_{\text {in }}+\frac{\lambda}{\Lambda}
$$

Equation (2)

The results are shown in Figure 8, where the effective refractive index calculated for all the laser beams are plotted against the applied potential. It is clear that $N_{\text {eff }}$ of the IOW device remains constant when different potential steps are applied to the ultra-thin ITO film.

In addition to the experimental results described above, we note that the thickness of the ITO film used on the IOW device is only $13 \mathrm{~nm}$, and even if the applied potential induces changes in the real part of the refractive index of the ITO film, those effects would have negligible effect on the effective refractive index of the whole IOW device and the coupling conditions would remain unchanged during potential modulations.

These results strongly suggest that the dominant effect for changes in the out-coupled optical power under potential modulations is originated in changes of the extinction coefficient of the ITO (imaginary part of the refractive index). Those changes impact the propagation loss (transmission) and consequently the out-coupled power.

The consistency of this conclusion is further corroborated by comparing the results of the two previous experiments. The normalized transmittance was calculated for the data shown in Figure 7 (for the 514 and $594 \mathrm{~nm}$ ) and compared to the equivalent quantity obtained under CV potential scans shown in Figure 6. For those calculations, displayed in Figure 9, we used the 
angle providing the highest out-coupled intensity at the open circuit potential for each potential step because when the data for $\mathrm{CV}$ potential scan was collected with the fixed incident angle, the light coupling condition was optimized at the open circuit potential. The comparisons of the normalized transmittance from these two potential modulation techniques for both the $594 \mathrm{~nm}$ and $514 \mathrm{~nm}$ are shown in Figure 9, where we can observe a good agreement for the independent approaches. Similar comparisons from the other laser lines (data not shown here) also provided good consistent for the measured data obtained by these two types of potential modulations.

\subsection{Alternating current potential modulation}

Optical measurements with an alternating current (AC) potential modulation applied to the ultra-thin ITO film were also investigated to examine the optical behavior of such structure at different modulation frequencies. For the probing light source, a super continuum fiber laser source (Fianium, SC-400-4) was used. An acousto-optical filter was connected to the fiber laser source to select a specific wavelength with a bandwidth of about $3 \mathrm{~nm}$. The out-coupled intensity of the guided light was collected by a PMT (Hamamatsu R928) and amplified by a current preamplifier (Stanford Research Systems, SR 570). The AC modulated applied potential and optical signals were then collected simultaneously by an oscilloscope (Agilent, DSO8104A Infiniium). A set of AC impedance potential modulations were applied, where each modulation had a fixed DC bias potential $(-0.2 \mathrm{~V}$ to $+0.28 \mathrm{~V})$, a constant $\mathrm{AC}$ amplitude $(10 \mathrm{mV})$, and a sequence of oscillation frequencies $(1 \mathrm{~Hz}$ to $20 \mathrm{~Hz})$.

The expression of the AC impedance potential modulation is shown in Equation 3, where $\boldsymbol{E}_{\boldsymbol{d} \boldsymbol{c}}$ is the potential DC bias, $\Delta \boldsymbol{E}_{\boldsymbol{a c}}$ is the AC amplitude, and $\boldsymbol{\omega}$ is the angular frequency of the potential oscillation. Under the condition that the AC amplitude is small enough, the optical 
response can be expressed with a linear approximation, as in Equation 4, where $\boldsymbol{I}_{\boldsymbol{d} \boldsymbol{c}}$ is the DC term, $\Delta \boldsymbol{I}_{\boldsymbol{a c}, \boldsymbol{R} \boldsymbol{e}}$ and $\Delta \boldsymbol{I}_{\boldsymbol{a c}, \boldsymbol{I m} \boldsymbol{m}}$ are the real and imaginary components (respectively) of the modulated optical AC term which include phase delay component.

$$
\begin{array}{ll}
E(t)=E_{d c}+\Delta E_{a c} \sin (\omega t) & \text { Equation (3) } \\
I(t, \lambda)=I_{d c}+\Delta I_{a c, R e} \sin (\omega t)+\Delta I_{a c, I m} \cos (\omega t) & \text { Equation (4) }
\end{array}
$$

The AC components of the optical response in the complex plane (where the real and imaginary components are plotted) are shown in Figure 10 for (a) $550 \mathrm{~nm}$ and (b) $580 \mathrm{~nm}$. The potential oscillation frequency has a clockwise direction from $1 \mathrm{~Hz}$ to $20 \mathrm{~Hz}$, as indicated by the black arrows. For the case of the 550-nm probing wavelength, the trace (which is mainly in fourth quadrant) shrinks ( $\underline{\Delta I}_{a \in}$ decreases) as the potential DC bias increases from $-0.2 \mathrm{~V}$ to +0.1 $\mathrm{V}$ and then expands again (mainly in the second quadrant). For the $580 \mathrm{~nm}$ wavelength, the optical response is confined to the fourth quadrant under the same interrogation conditions. Those results highlight that both the modulation frequency and the DC bias have an important impact on the optical response of the ITO film. And again, those behaviors are highly dependent on the wavelength under consideration.

The potential DC bias can be interpreted as a constant potential step or the potential point at the slow CV scans. The DC components of the optical signals were compared to the optical response under $\mathrm{CV}$ potential scan. The normalized transmittance curves are shown in Figure 11 for (a) $550 \mathrm{~nm}$ and (b) $580 \mathrm{~nm}$ separately. For the CV scan, the normalized 
transmittance was calculated in the potential range from $-0.2 \mathrm{~V}$ to $+0.28 \mathrm{~V}$. Good consistent are observed for both probing wavelengths.

\section{Discussions}

Our data shows that when an ultra-thin ITO film undergoes an electric potential modulation, the out-coupled intensity of a propagating light beam inside the waveguide (which incorporates the ITO film) changes and different probing wavelengths have different profiles. From the experiments under CV scans, potential steps, and AC potential modulations, we were able to conclude that the extinction coefficient (absorption) of the ultra-thin ITO plays a dominant role and has a discernible dependence on the applied potential, and this dependence varies for different wavelengths in the spectral region of the measured data.

As a semiconductor material, the energy band structures of the ITO material have been extensively studied [7]. The optical properties are known to be related to the energy band structures, which are determined by the ratios of the chemical elements indium, tin, and oxygen that form the film. And especially in the visible region, the band structure is dependent on the doping of the tin atom. This phenomenon suggests that the extinction coefficient of ITO film is strongly related to the oxygen vacancy concentration. When a potential modulation is applied to an ultra-thin ITO film, oxygen vacancy concentration could be modified and those vacancies could be changed by Sn element or $\mathrm{H}+$ ion diffusion into the film.

The relatively thick ITO films $(400 \mathrm{~nm})$ were also tested with acid solution under the similar CV potential scans. With low $\mathrm{pH}$ values 4.5 of the electrolyte solution, ITO samples with low $\mathrm{O}_{2}$ gas flow rate during deposition $(0.55 \mathrm{sccm}$ and $0.6 \mathrm{sccm})$ were darken in the area immersed in the solution, as shown in Figure 12. However, ITO samples with higher oxygen 
content $\left(0.7 \mathrm{sccm} \mathrm{O}_{2}\right.$ flow rate) were not darkened. This observation further emphasizes the role of the oxygen concentration in the optical properties of the ITO film. Work has been reported with extremely high electrical field on the thick ITO films. With the negative potential, the film was darkened with lower oxygen content, while at positive potential the film resulted in higher oxygen concentration [32].

The spectroscopic properties of the relatively thick ITO films (400 nm) were also tested in the same buffer solution and identical $\mathrm{CV}$ potential scans using a UV-Visible spectrophotometer with single transmission technique. No significant change in the transmitted intensity could be detected for these thick films. The reason could be that transmittance measurement is not sensitive enough to show the change from the films since the optical probing length was limited to the thickness of the ITO films or the potential modulation applied on these thick ITO films do not have the same effect as in the ultra-thin ITO films.

\section{Conclusions}

By exploiting the high sensitivity provided by a single-mode integrated optical waveguide platform, the spectroscopic properties of an ultra-thin (about $13 \mathrm{~nm}$ ) ITO film deposited on this photonic device were investigated under different potential modulation schemes in the visible spectral region. We confirm through those independent experimental approaches that such thin ITO film exhibits unique spectroscopic features under potential modulations. And those features can be highly relevant and must be taken into consideration in many applications. For example, one area with important applications of the ultra-thin transparent and conductive ITO electrode is for the spectroelectrochemical studies of redox reactions of surface-confined species. The ultra-thin ITO film incorporated into the single-mode 
integrated optical waveguide has already been demonstrated in redox reaction investigations of a protein layer of cytochrome-c molecules [23]. When a proper analysis of the ITO features discussed here were taken into consideration, experimental data taken under AC potential modulation allowed us to optically retrieve accurate information for the electron transfer rate of this protein assembly adsorbed onto the ITO electro-active interface. And because of the high sensitivity of the single-mode integrated optical waveguide and the high quality of the ultra-thin

ITO film, as low as $10^{-14} \mathrm{~mol} / \mathrm{cm}^{2}$ surface coverage of cytochrome-c proteins were detected under a small $(15 \mathrm{mV})$ amplitude of an $\mathrm{AC}$ modulation of the applied electric potential. A multitude of applications based on this electro-active single-mode integrated optical waveguide technology is envisioned for implementation of advanced chemical- and bio-sensing devices and electro-optical characterization of novel solar cells materials.

\section{Acknowledgements:}

The authors acknowledge financial support from National Institute of Health (NIH award RR022864), National Science Foundation (NSF EPSCoR award 0814194), and Kentucky Science and Engineering Foundation (KSEF award 1869-RDE-012). 


\section{References:}

[1] J. Pláa, M. Tamasia, R. Rizzolib, M. Losurdoc, E. Centurionib, C. Summonteb, F. Rubinellid, Optimization of ITO layers for applications in a-Si/c-Si heterojunction solar cells. Thin Solid Films, 425 (2003), 185-192.

[2] Supachai Ngamsinlapasathiana, Thammanoon Sreethawongb, Yoshikazu Suzukia, Susumu Yoshikawaa, Doubled layered ITO/SnO2 conducting glass for substrate of dye-sensitized solar cells. Solar Energy Materials and Solar Cells, 90 (2006), 2129-2140.

[3] Jin-A Jeong, Han-Ki Kim, Low resistance and highly transparent ITO-Ag-ITO multilayer electrode using surface plasmon resonance of Ag layer for bulk-heterojunction organic solar cells. Solar Energy Materials and Solar Cells, 93 (2009), 1801-1809.

[4] M. Fahland, P.K., C. Charton, Low resisitivity transparent electrodes for displays on polymer substrates. Thin Solid Films, 392 (2001), 334-337.

[5] U. Betz, M. Kharrazi Olsson, J. Marthy, M.F. Escolá, F. Atamny, Thin films engineering of indium tin oxide: Large area flat panel displays application. Surface and Coatings Technology, 200 (2006), 57515759.

[6] Granqvist, C.G., Transparent conductive electrodes for electrochromic devices a review. Applied Physics A, 57 (1993), 19-24.

[7] C.G. Granqvist, and A. Hultaker, Transparent and conducting ITO films new developments and applications. Thin Solid Films, 411 (2002), 1-5.

[8] Yung-Chi Yao, Meng-Tsan Tsai, Hsu-Cheng Hsu, Li-Wei She, Chun-Mao Cheng, Chien-Jang Wu, Yi-Ching Chen, and Ya-Ju Lee, Use of two-dimensional nanorod arrays with slanted ITO film to enhance optical absorption for photovoltaic applications. Optics Express, 20 (2012), 3479-3489.

[9] Arunandan Kumar, R. Srivastava, M. N. Kamalasanan, and Dalip Singh Mehta, Enhancement of light extraction efficiency of organic light emitting diodes using nanostructured indium tin oxide. Opitcs Letters, 37 (2012), 575-577. 
[10] J. S. Kim, M. Granström, R. H. Friend, N. Johansson, W. R. Salaneck, R. Daik, W. J. Feast and F. Cacialli, Indium-tin oxide treatments for single- and double-layer polymeric light-emitting diodes: The relation between the anode physical, chemical, and morphological properties and the device performance. Journal of Applied Physics, 84 (1998), 6859-6870.

[11] Anand Jaina, Giulio Gazzolaa, Aurora Panzerab, Michele Zanonic, Enrico Marsili, Visible spectroelectrochemical characterization of Geobacter sulfurreducens biofilms on optically transparent indium tin oxide electrode. Electrochimica Acta, 56 (2011), 10776-10785.

[12] Ge C, Doherty WJ 3rd, Mendes SB, Armstrong NR, Saavedra SS., Voltammetric and waveguide spectroelectrochemical characterization of ultrathin poly(aniline)/poly(acrylic acid) films self-assembled on indium-tin oxide. Talanta, 65 (2005), 1126-31.

[13] Chih-Hao Lianga, Sheng-Chau Chena, Xiaoding Qib, Chi-San Chena, Chih-Chao Yang, Influence of film thickness on the texture, morphology and electro-optical properties of indium tin oxide films. Thin Solid Films, 519 (2010), 345-350.

[14] F. Wanga, M.Z. Wu, Y.Y. Wang, Y.M. Yu, X.M. Wu, L.J. Zhuge, Influence of thickness and annealing temperature on the electrical, optical and structural properties of AZO thin films. Vacuum, 89 (2013), 127-131.

[15] T.C. Gorjanca, D.Leong., C. Py , D. Roth, Room temperature deposition of ITO using r.f. magnetron sputtering. Thin Solid Films, 413 (2002), 181-185.

[16] Dong-Ho Kim, Mi-Rang Park, Hak-Jun Lee, Gun-Hwan Lee, Thickness dependence of electrical properties of ITO film deposited on a plastic substrate by RF magnetron sputtering. Applied Surface Science, 253 (2006), 409-411.

[17] A.L. Swint and P.W. Bohn, Effect of the interfacial chemical environment on in-plane electronic conduction of indium tin oxide role of surface charge dipole magnitude, and carrier injection, Langmuir, 20 (2004), 4076-84.

[18] Matveeva, E., Electrochemistry of the indium-tin oxide electrode in $1 \mathrm{M} \mathrm{NaOH}$ electrolyte. Journal of The Electrochemical Society, 152 (2005), H138-H145. 
[19] Dai Kato, G.Xue, Yuzuru Iwasaki, Yoshiki Hirata, Ryoji Kurita, and Osamu Niwa, Heavy phosphate adsorption on amorphous ITO film electrodes nano-barrier effect for highly selective exclusion of anionic species. Langmuir, 23 (2007), 8400-5.

[20] F. Nüesch, E. W. Forsythe, Q. T. Le, Y. Gao and L. J. Rothberg, Importance of indium tin oxide surface acido basicity for charge injection into organic materials based light emitting diodes. Journal of Applied Physics, 87 (2000), 7973-7980.

[21] Er-Jia Guo, Haizhong Guo, Huibin Lu, Kuijuan Jin, Meng He and Guozhen Yang, Structure and characteristics of ultrathin indium tin oxide films. Applied Physics Letters, 98 (2011), 011905-3.

[22] S. Stankowski and J.J. Ramsden, Voltage-dependent coupling of light into ITO-covered waveguides. Journal of Physics D: Applied Physics, 35 (2002), 299-304.

[23] L.R. Cruza, C. Legnania, I.G. Matosoa, C.L. Ferreiraa, and H.R. Moutinho, Influence of pressure and annealing on the microstructural and electro-optical properties of RF magnetron sputtered ITO thin films. Materials Research Bulletin, 39 (2004), 993-1003.

[24] R. Swanepoel, Determination of the thickness and optical constants of amorphous silicon. Journal of Physics E: Scientific Instruments, 16 (1983), 1214-1222.

[25] D. R. Dunphy, S.B.Mendes, S. S. Saavedra, and Neal R. Armstrong, The Electroactive integrated optical waveguide ultrasensitive spectroelectrochemistry of submonolayer adsorbates. Analytical chemistry, 69 (1997), 3086-3094.

[26] D. Mergel, and Z. Qiao, Correlation of lattice distortion with optical and electrical properties of

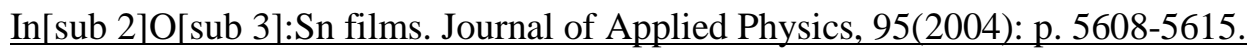
[27] John Thomas Bradshaw, S.B.Mendes, Neal R. Armstrong, and S. Scott Saavedra, Broadband coupling into a single-mode, electroactive integrated optical waveguide for spectroelectrochemical analysis of surface-confined redox couples. Analytical chemistry, 75 (2003), 1080-1088.

[28] R. S. Wiederkehr, G.C. Hoops, M.M. Aslan, C.L. Byard, and S.B. Mendes, Investigations on the Q and CT bands of cytochrome c submonolayer adsorbed on an alumina surface using broadband 
spectroscopy with single-mode integrated optical waveguides. The Journal of Physical Chemistry C, 113 (2009), 8306-8312.

[29] Rodrigo S. Wiederkehr, H., Geoffrey C., Sergio B. Mendes, Effects of sodium chloride on the properties of chlorophyll a submonolayer adsorbed onto hydrophobic and hydrophilic surfaces using broadband spectroscopy with single-mode integrated optical waveguides. Optical Engineering, 50 (2011), 071109-8.

[30] M.B.Pereira, J.S. Craven, and S.B. Mendes, Solid immersion lens at the aplanatic condition for enhancing the spectral bandwidth of a waveguide grating coupler. Optical Engineering, 49 (2010), 124601-8.

[31] J. He, M.Lu, X. Zhou, J.R. Cao, K.L. Wang, L.S. Liao, Z.B. Deng, X.M. Ding, X.Y. Hou, S.T. Lee, Damage study of ITO under high electric field. Thin solid films, 363 (2000), 240-243.

[32] X. Han, and S.B. Mendes, Optical impedance spectroscopy with single-mode electro-activeintegrated optical waveguides. Analytical chemistry, 86 (2014), 1468-77. 


\section{List of figures:}

Figure 1: Effects of oxygen flow rate during the sputtering deposition process on the optical and electrical properties of the ITO film with data provided for measurements before and after an inert annealing treatment. Experimental data of (a) the extinction coefficient (imaginary part of the refractive index) at $550 \mathrm{~nm}$, and (b) the electrical resistivity. As the resistivity before the annealing process is very high at increased levels of $\mathrm{O} 2$ flow rate, the measured resistivity data at $0.70 \mathrm{sccm}(2.7 \mathrm{ohm} \mathrm{cm})$ and $0.80 \mathrm{sccm}$ (not conductive) are not displayed in Fig. 1b.

Figure 2: Schematic structure of the single-mode integrated optical waveguide with an over-layer of an ultra-thin ITO film under investigation. An actual IOW device with broadband light dispersion of the out-coupled guided optical beam is shown in the inset.

Figure 3: Power spectrum of the broadband out-coupled guided light at different values of the applied potential during a particular CV scan.

Figure 4: Normalized transmittance for 3-folded cycles of CV scan obtained from broadband spectrum of Figure 3. Traces of the transmittance show a clear dependence on the applied potential, and that dependence changes for different wavelengths. For clarity, each curve is shifted by one division. Figure 5: Normalized transmittance against time for several laser lines under CV potential scan. For clarity each transmission curve is shifted by one division. The trace of the potential modulation is also displayed as the black curve, with the potential ranging from $-0.4 \mathrm{~V}$ to $+0.8 \mathrm{~V}$.

Figure 6: Normalized transmittance over 5-folded cycles of the applied potential is plotted for each laser line. The results with the laser lines are consistent with the data obtained with the broadband light source (Figure 3 and 4) and show again the high repeatability of the spectroscopic features during the potential scans. For clarity, each curve is shifted by one division.

Figure 7: Out-coupled intensity against the input angle for two wavelengths: (a) $594 \mathrm{~nm}$ and (b) $514 \mathrm{~nm}$. Measurements were performed at several potential values applied to the ITO film. Not all experimental results (at potential steps $-0.2 \mathrm{~V}, 0.2 \mathrm{~V}, 0.6 \mathrm{~V}$ and open circuit condition) are shown here for the clarity. 
Figure 8: Effective refractive index for each laser beams at each applied potential step.

Figure 9: Normalized transmittance comparison between CV potential scan and potential steps from 594 $\mathrm{nm}$ laser line (a) and $514 \mathrm{~nm}$ laser line (b). Red solid lines are from CV potential scans, and blue diamonds are from discrete applied potentials.

Figure 10: Complex plane representation of the optical response for an AC-modulation of the applied potential to the ultra-thin ITO film at several potential DC biases for the (a) $550 \mathrm{~nm}$ and (b) $580 \mathrm{~nm}$ wavelengths. Modulation frequency increases clockwise from $1 \mathrm{~Hz}$ to $20 \mathrm{~Hz}$.

Figure 11: Comparison of normalized transmittance between the optical DC components from the AC impedance potential modulation and the $\mathrm{CV}$ potential scan used a tungsten-halogen white light source. A good agreement is observed for each probing wavelength (a) $550 \mathrm{~nm}$ and (b) $580 \mathrm{~nm}$.

Figure 12: Picture of a 400-nm thick ITO films under a CV potential modulation in $4.5 \mathrm{pH}$ acid solution. 


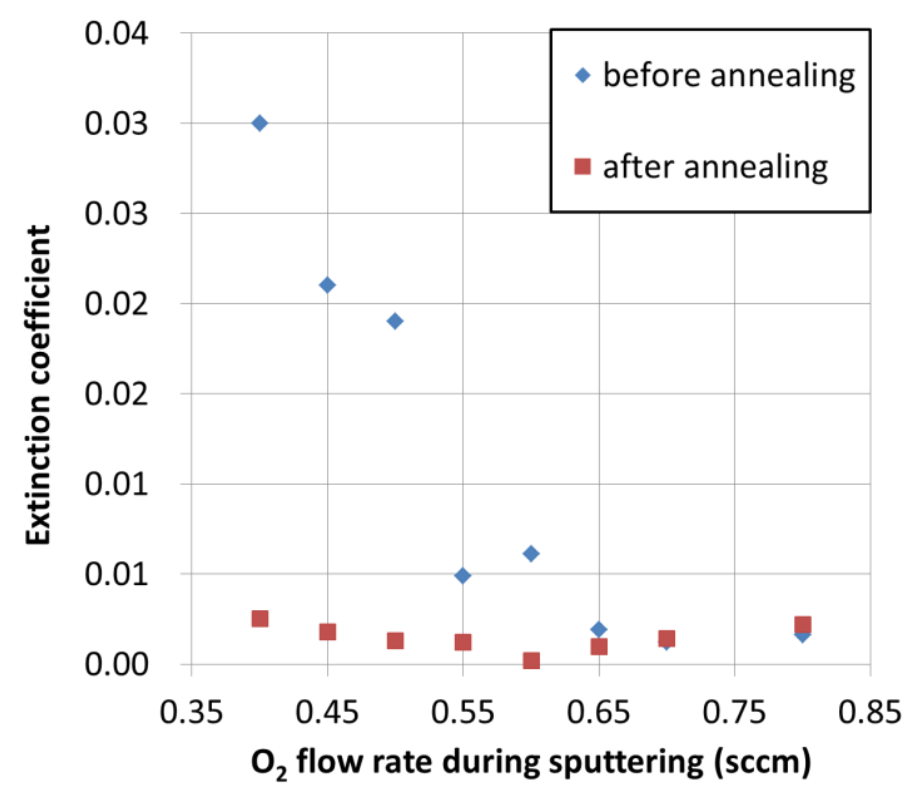

(a)

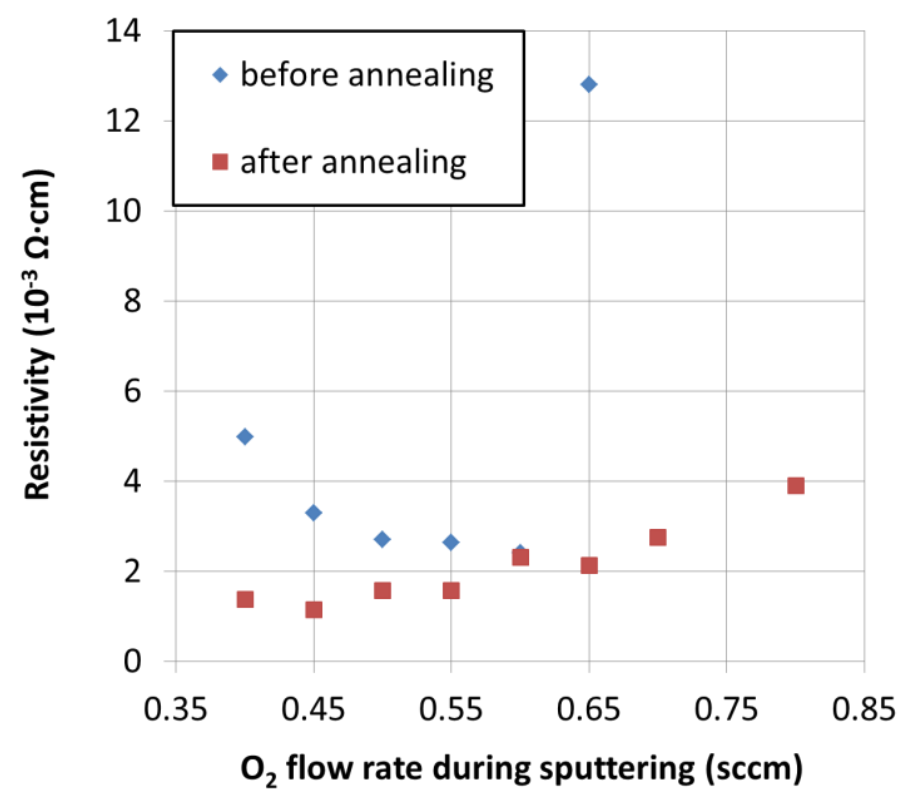

(b)

Figure 1: Effects of oxygen flow rate during the sputtering deposition process on the optical and electrical properties of the ITO film with data provided for measurements before and after an inert annealing treatment. Experimental data of (a) the extinction coefficient (imaginary part of the refractive index) at $550 \mathrm{~nm}$, and (b) the electrical resistivity. As the resistivity before the annealing process is very high at increased levels of $\mathrm{O}_{2}$ flow rate, the measured resistivity data at $0.70 \mathrm{sccm}$ (about $2.7 \mathrm{ohm} \mathrm{cm}$ ) and $0.80 \mathrm{sccm}$ (not conductive) are not displayed in Fig. 1b. 


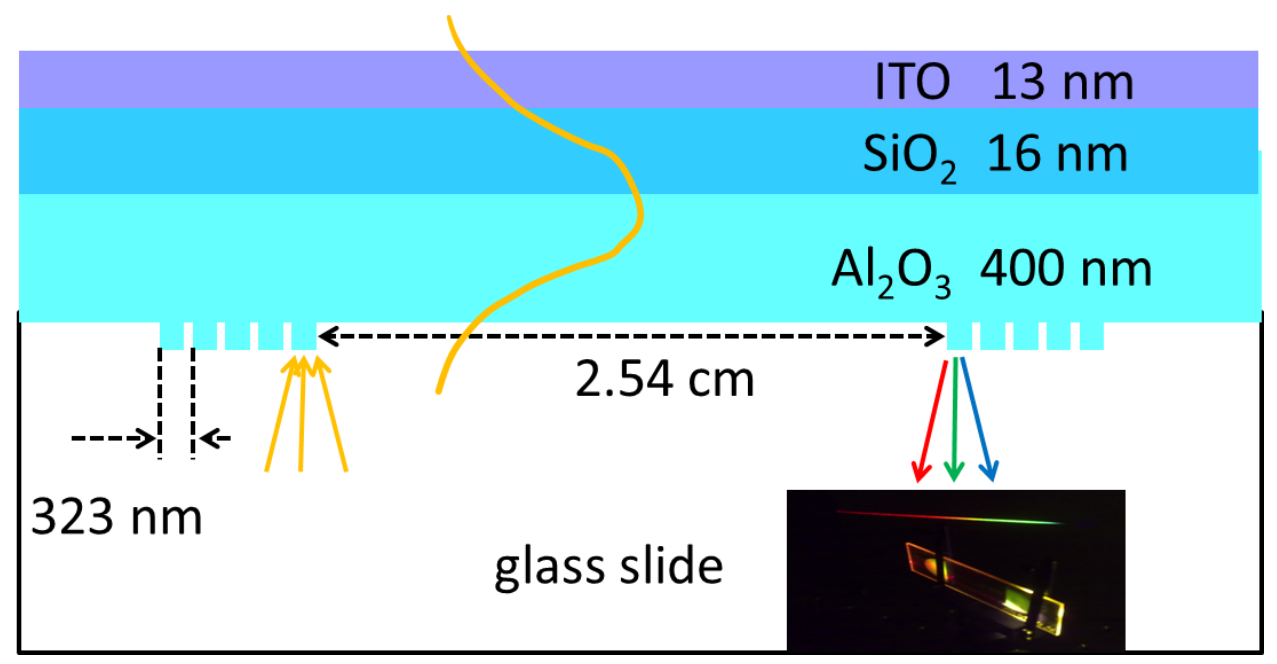

Figure 2: Schematic structure of the single-mode integrated optical waveguide with an over-layer of an ultra-thin ITO film under investigation. An actual IOW device with broadband light dispersion of the out-coupled guided optical beam is shown in the inset. 


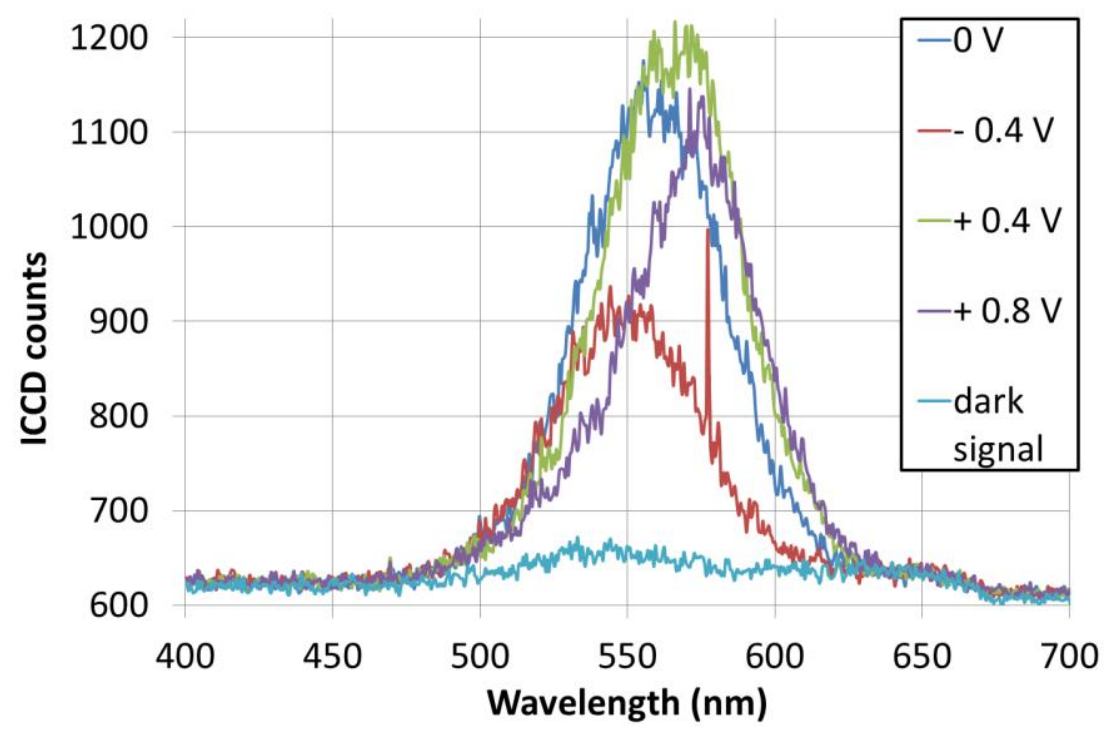

Figure 3: Power spectrum of the broadband out-coupled guided light at different values of the applied potential during a particular CV scan. 


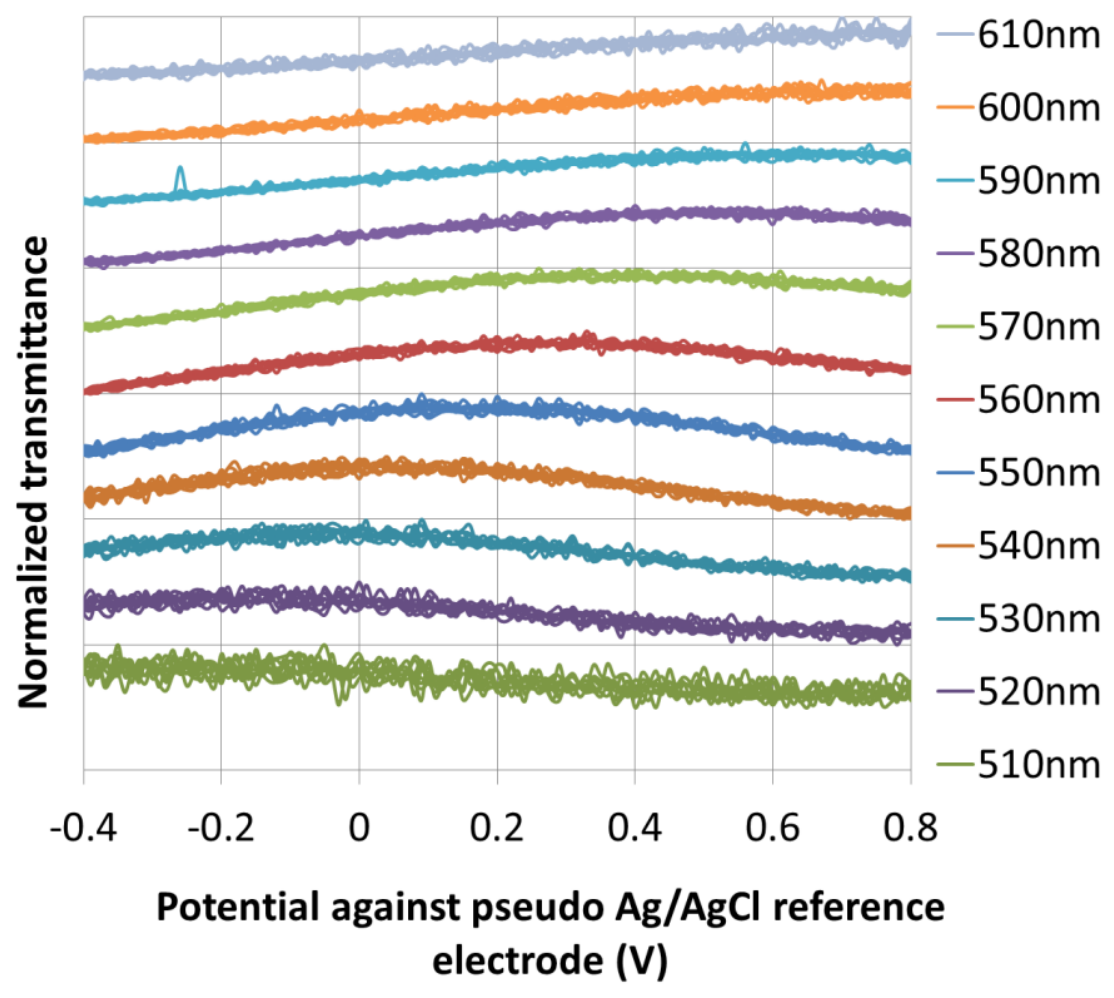

Figure 4: Normalized transmittance for 3-folded cycles of CV scan obtained from broadband spectrum of Figure 3. Traces of the transmittance show a clear dependence on the applied potential, and that dependence changes for different wavelengths. For clarity, each curve is shifted by one division. 


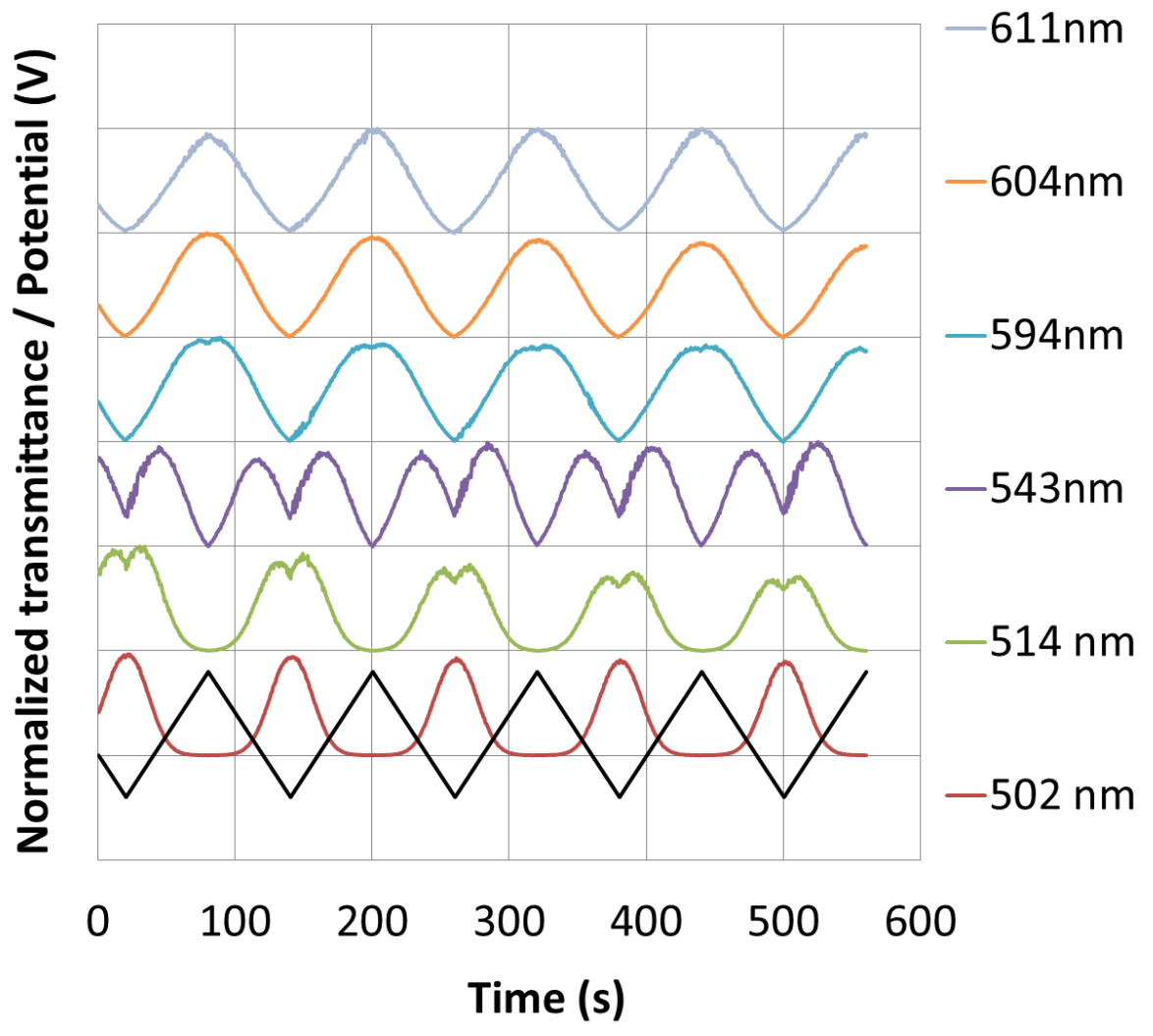

Figure 5: Normalized transmittance against time for several laser lines under $\mathrm{CV}$ potential scan. For clarity, each transmission curve is shifted by one division. The trace of the potential modulation is also displayed_with the potential ranging from $-0.4 \mathrm{~V}$ to $+0.8 \mathrm{~V}$. 


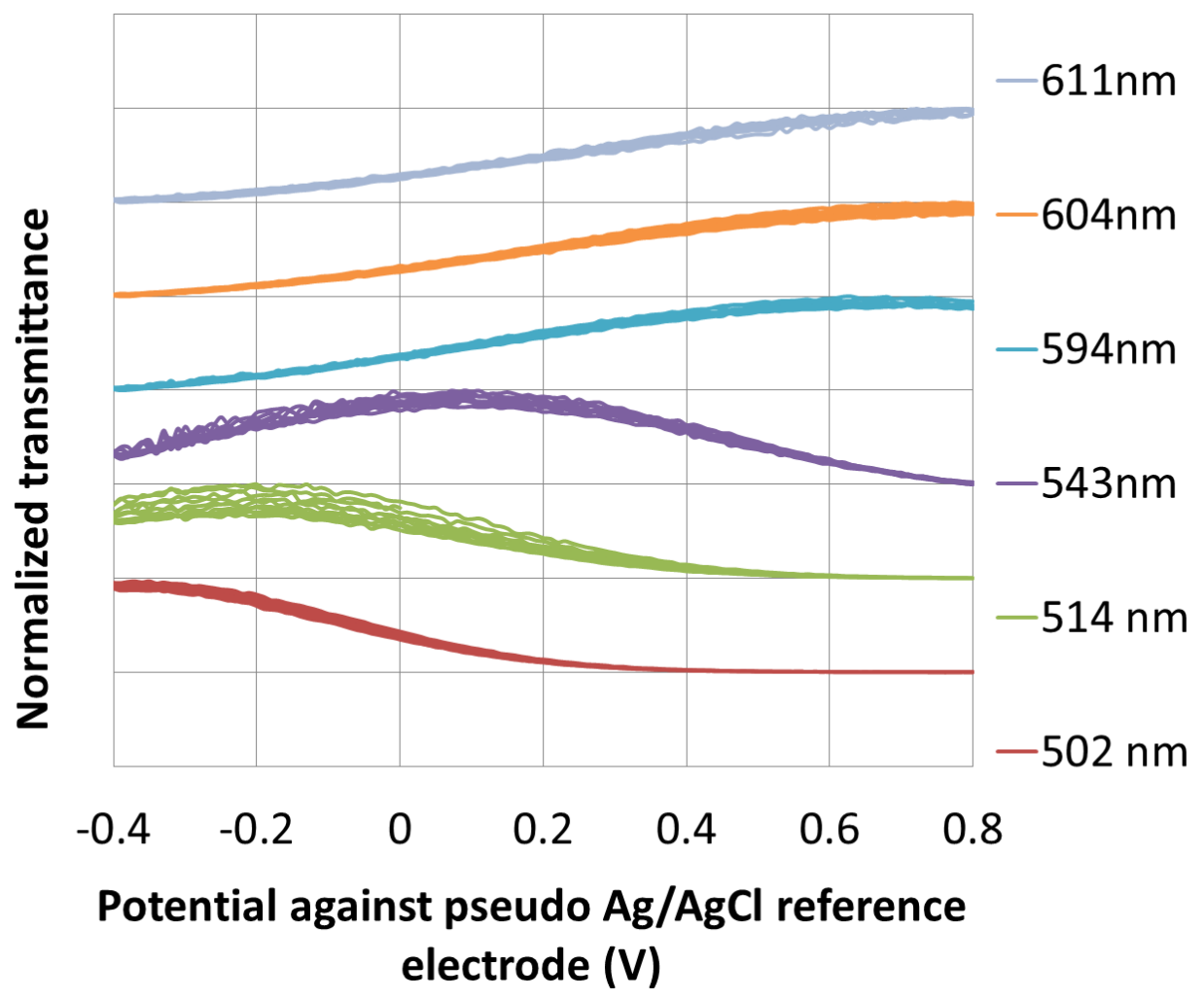

Figure 6: Normalized transmittance over 5-folded cycles of the applied potential is plotted for each laser line. The results with the laser lines are consistent with the data obtained with the broadband light source (Figure 3 and 4) and show again the high repeatability of the spectroscopic features during the potential scans. For clarity, each curve is shifted by one division. 


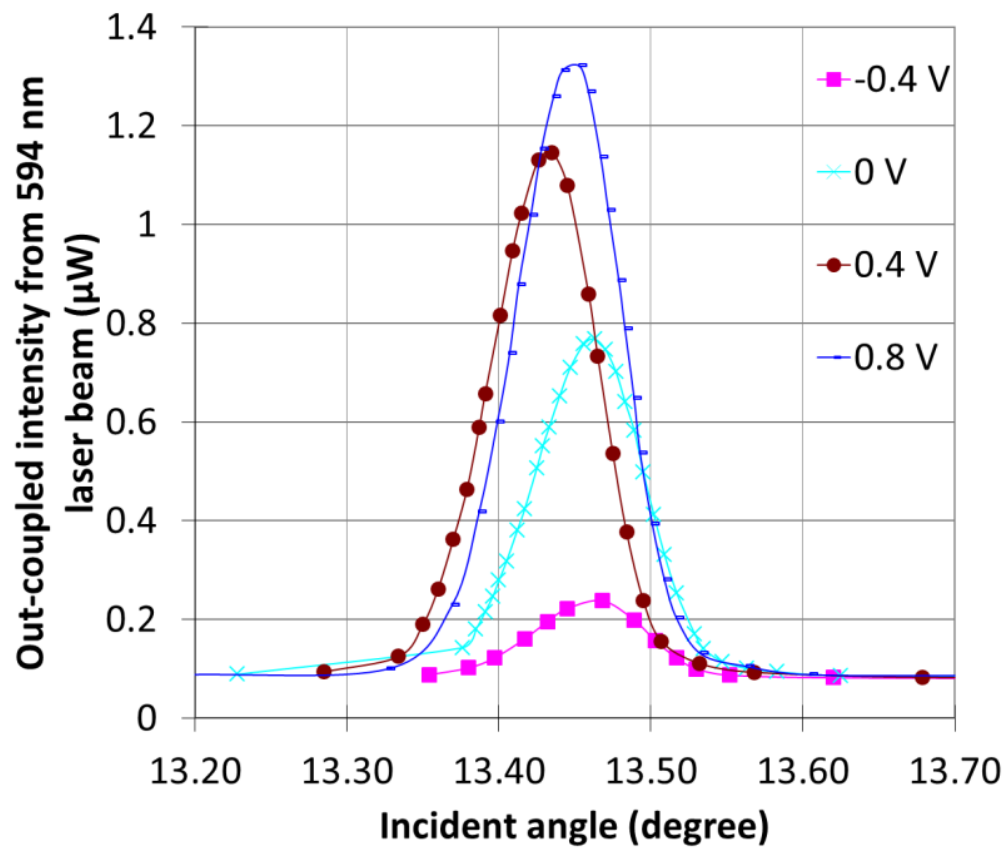

(a)

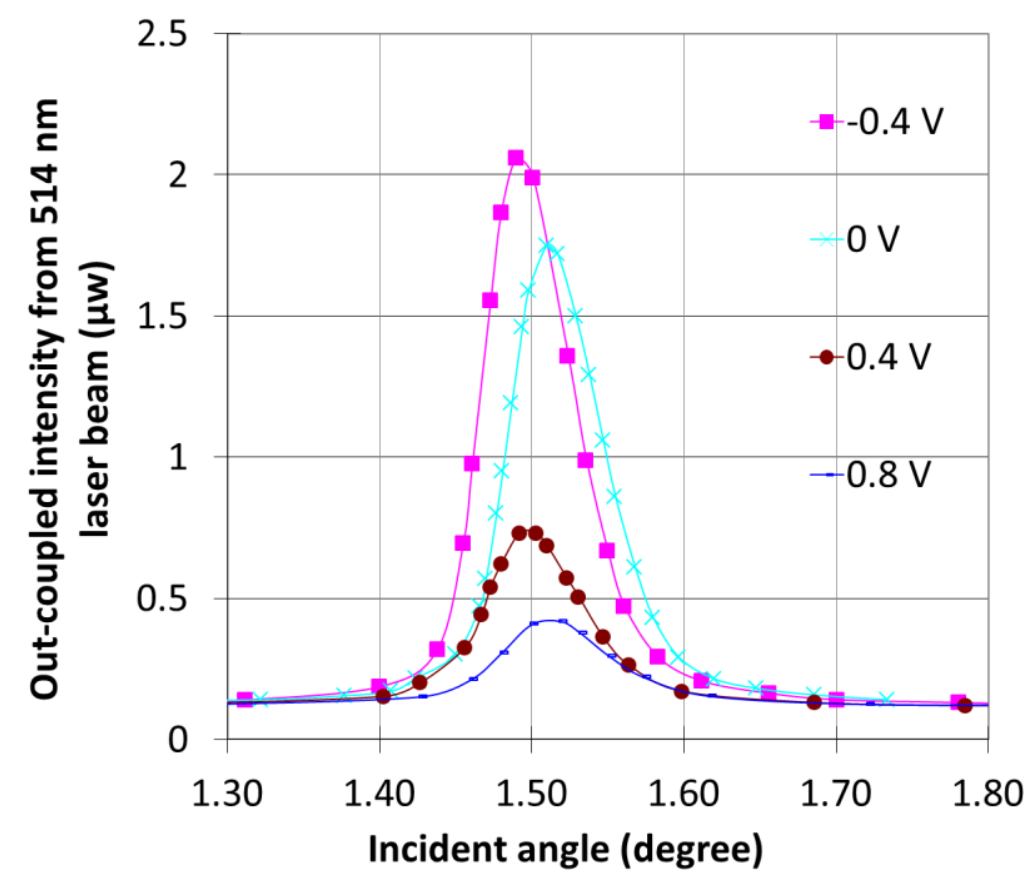

(b)

Figure 7: Out-coupled intensity against the input angle for two wavelengths: (a) $594 \mathrm{~nm}$ and (b) $514 \mathrm{~nm}$. Measurements were performed at several potential values applied to the ITO film. Not all experimental results (at potential steps $-0.2 \mathrm{~V}, 0.2 \mathrm{~V}, 0.6 \mathrm{~V}$ and open circuit condition) are shown here for the clarity. 


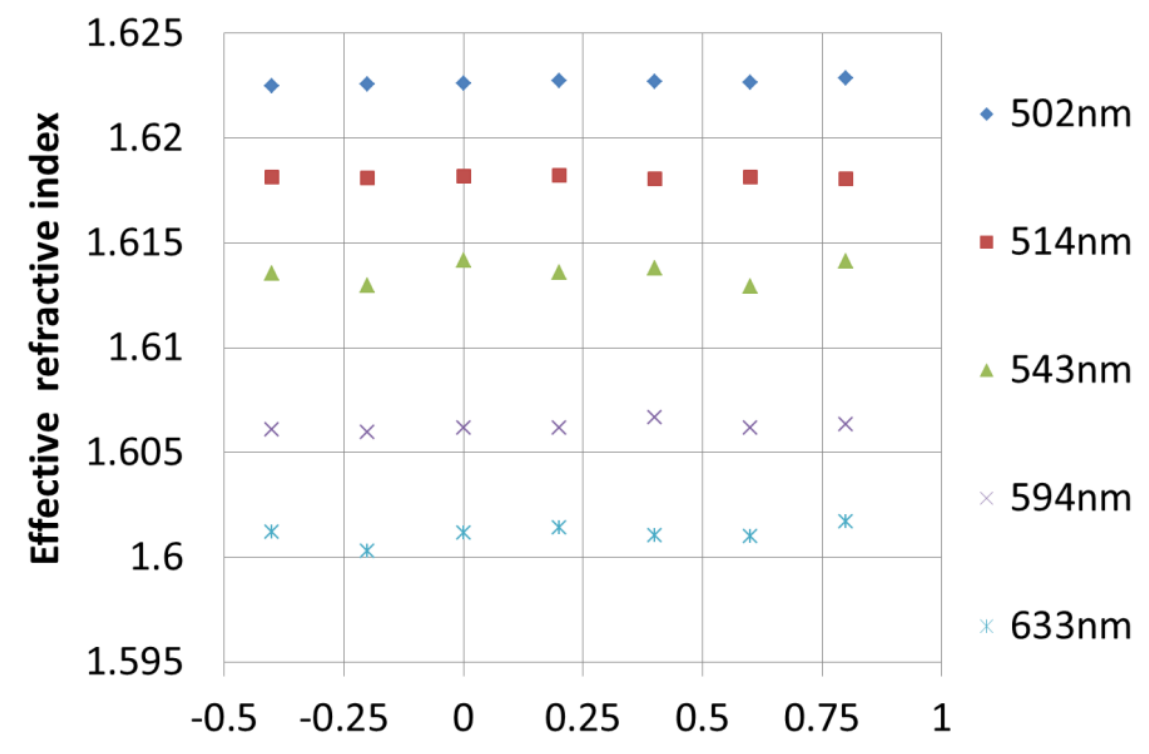

Potential applied against $\mathrm{Ag} / \mathrm{AgCl}$ reference electrode (V)

Figure 8: Effective refractive index for each laser beams at each applied potential step. 


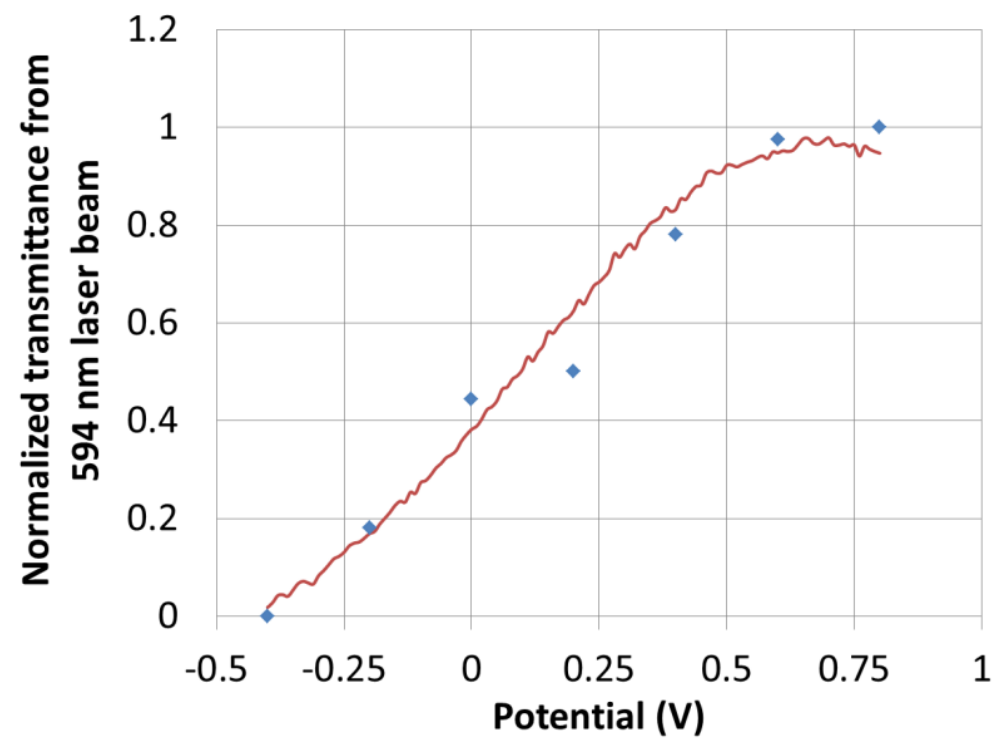

(a)

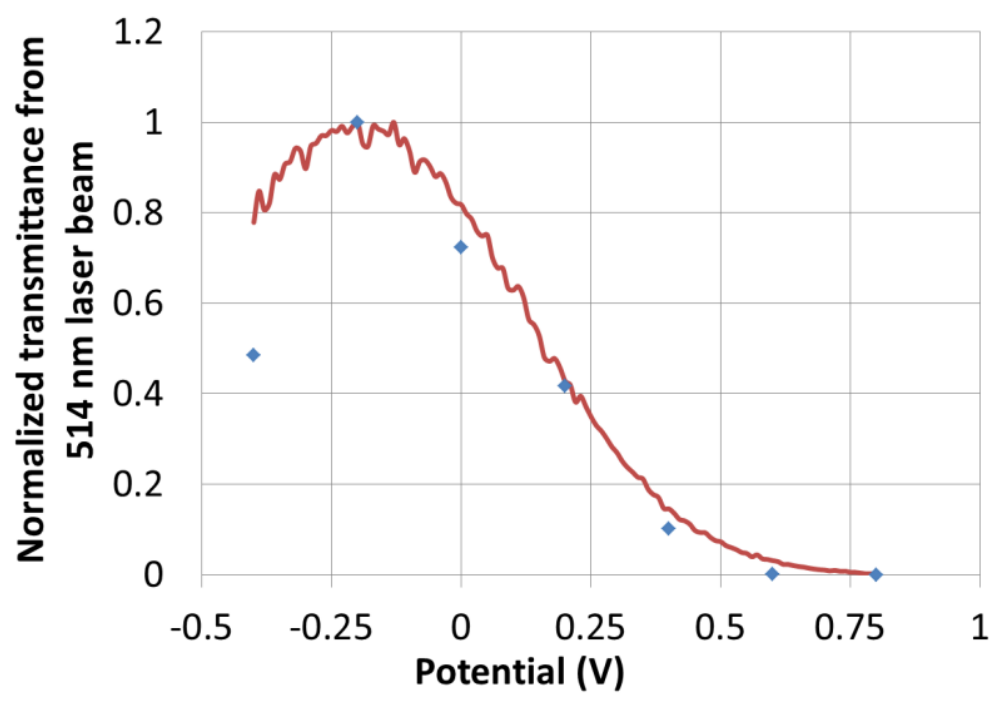

(b)

Figure 9: Normalized transmittance comparison between $\mathrm{CV}$ potential scan and potential steps from $594 \mathrm{~nm}$ laser line (a) and $514 \mathrm{~nm}$ laser line (b). Red solid lines are from CV potential scans, and blue diamonds are from discrete applied potentials. 


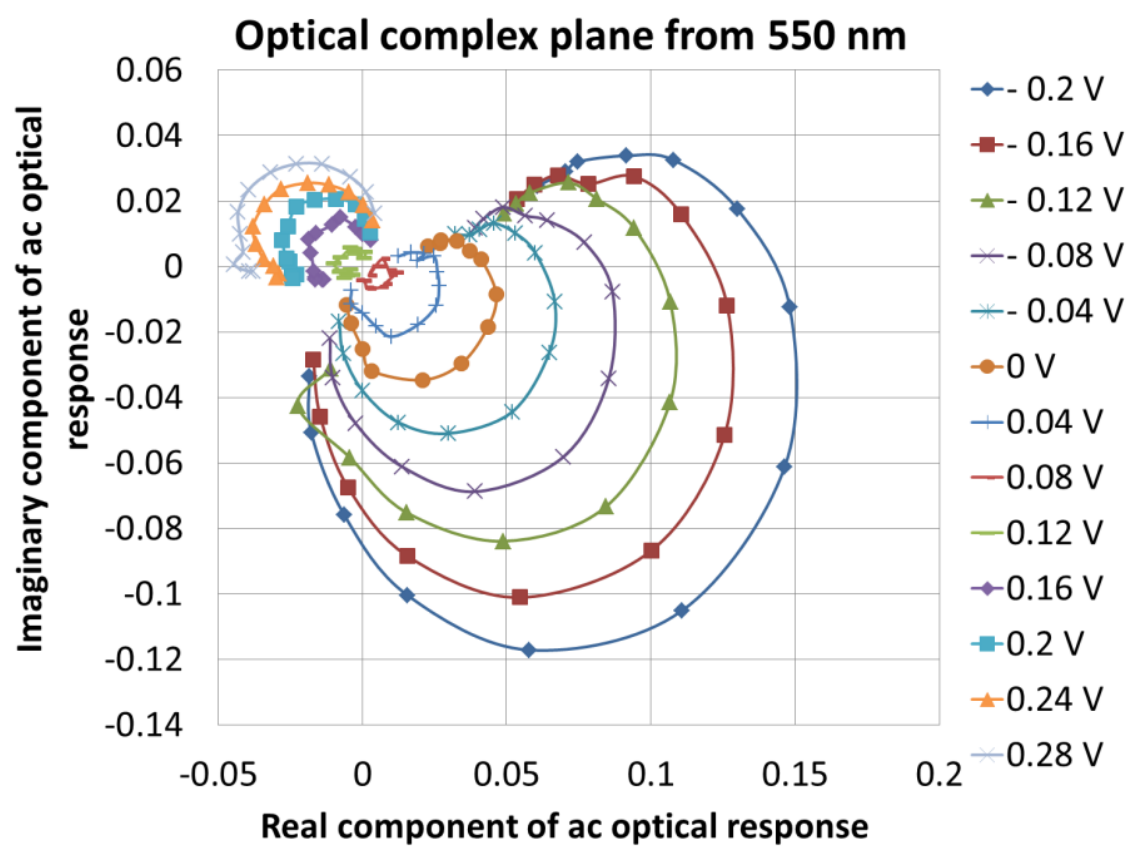

(a)

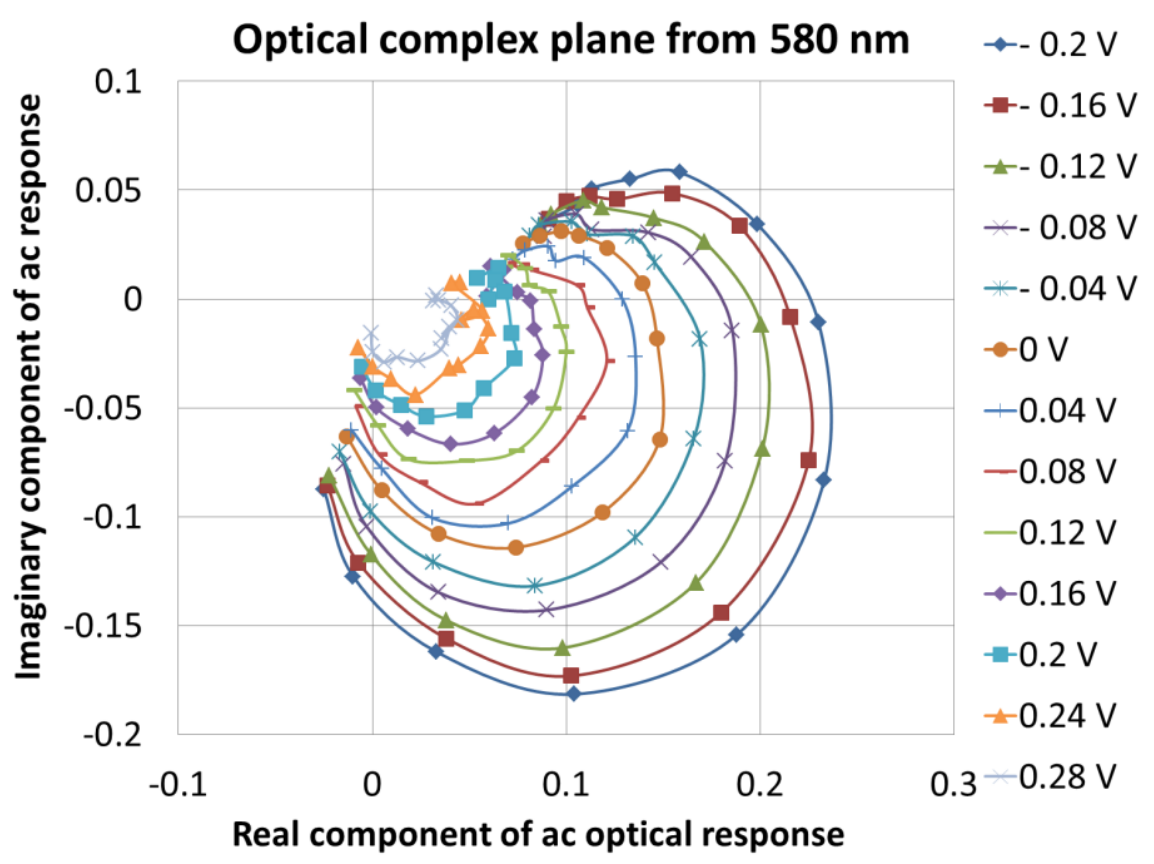

(b)

Figure 10: Complex plane representation of the optical response for an ACmodulation of the applied potential to the ultra-thin ITO film at several potential DC biases for the (a) $550 \mathrm{~nm}$ and (b) $580 \mathrm{~nm}$ wavelengths. Modulation frequency increases clockwise from $1 \mathrm{~Hz}$ to $20 \mathrm{~Hz}$. 


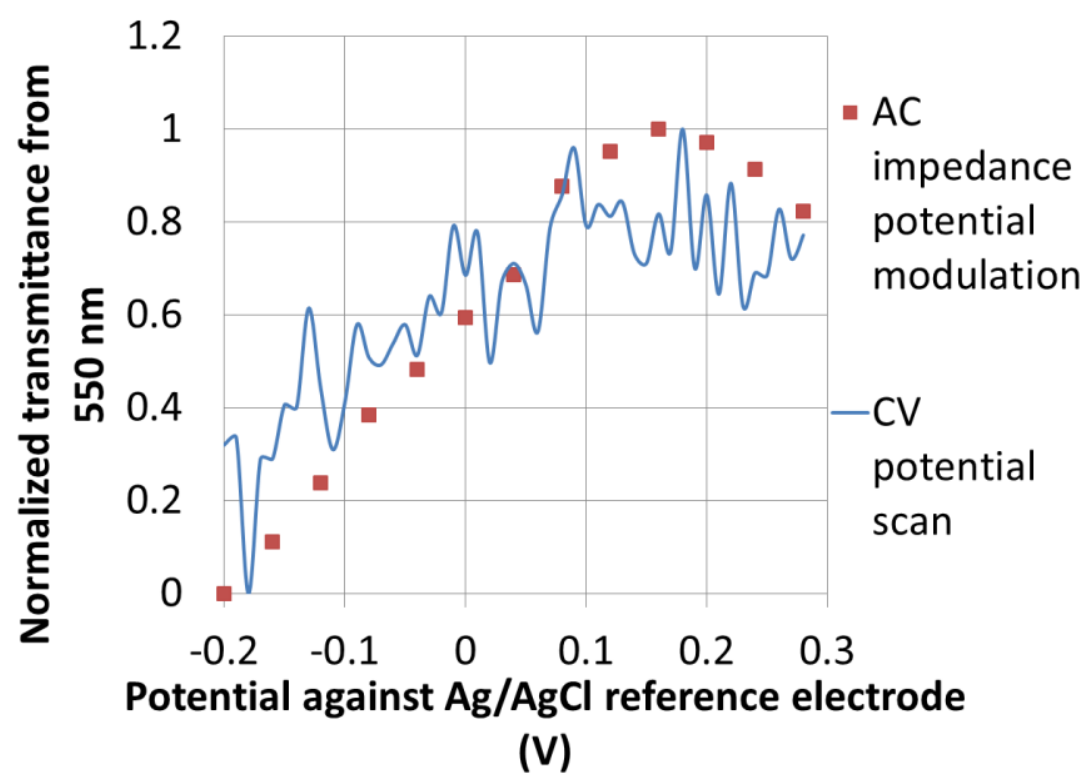

(a)

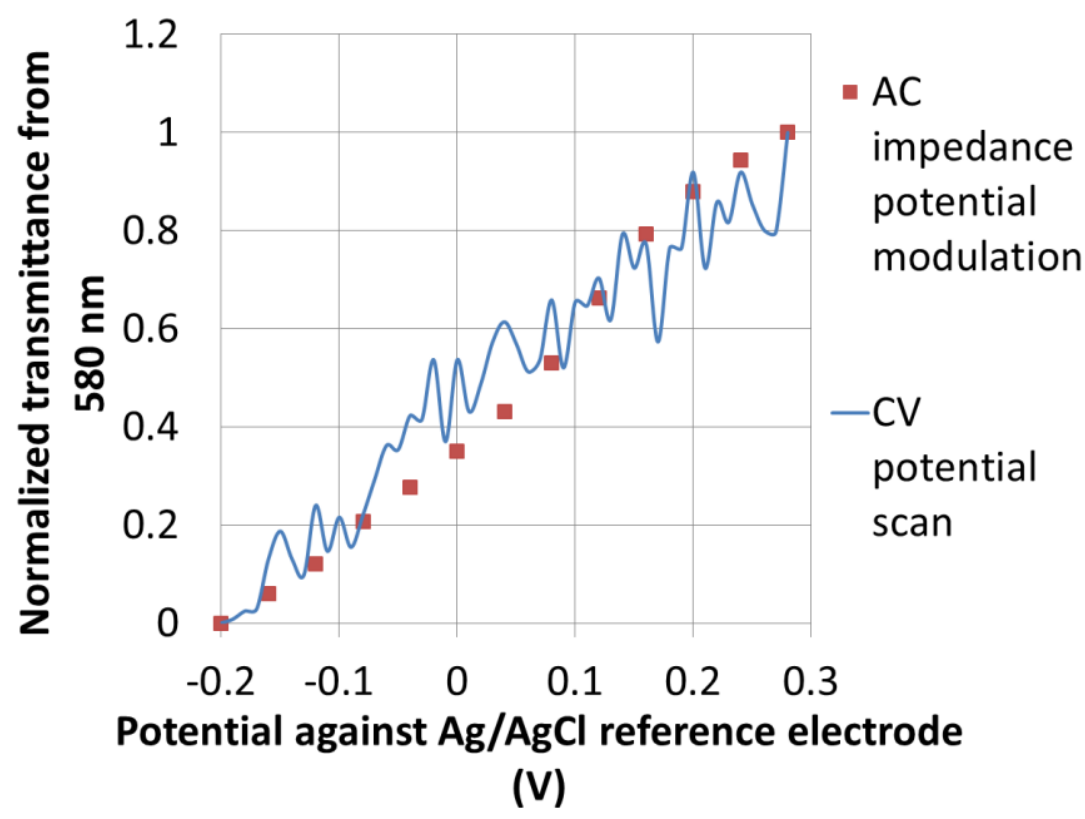

(b)

Figure 11: Comparison of normalized transmittance between the optical DC components from the $\mathrm{AC}$ impedance potential modulation and the $\mathrm{CV}$ potential scan used a tungsten-halogen white light source. A good agreement is observed for each probing wavelength (a) $550 \mathrm{~nm}$ and (b) 580 nm. 


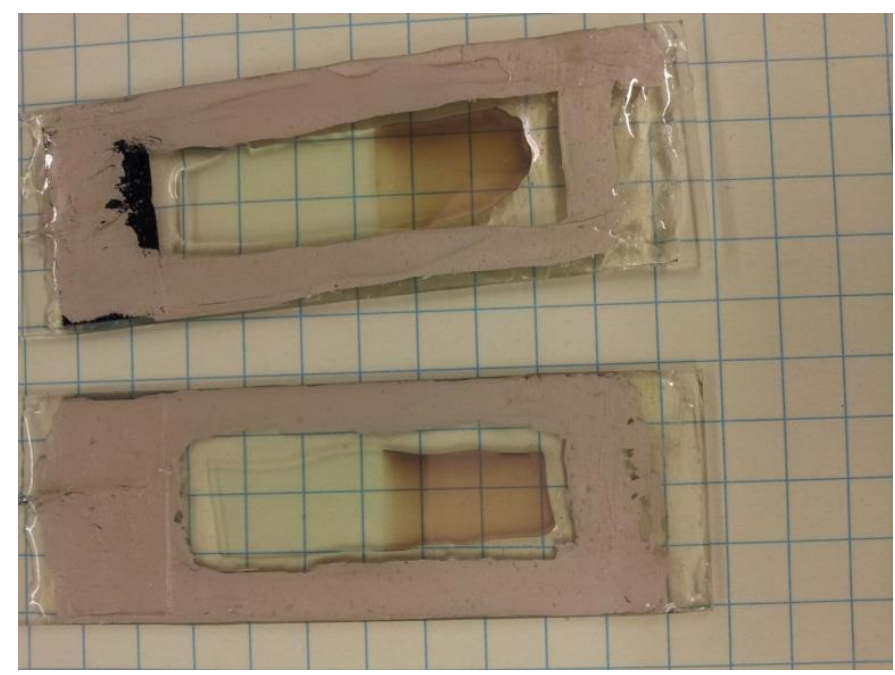

Figure 12: Picture of a 400-nm thick ITO films under a CV potential modulation in $4.5 \mathrm{pH}$ acid solution. 